\title{
The Prevalence of Children with a History of Sexual Abuse Hospitalized in the Psychiatric Setting
}

PATRICIA A. MORRIS, MS, RN, and SUZANNE M. BIHAN, BSN, RN

This descriptive study systematically sampled 100 closed records of children hospitalized at a children's psychiatric hospital in a midwest city over the past 5 years to determine the prevalence of children identified as having a history of sexual abuse. The symptoms of abuse manifested by these children were compared with the prevalence of children without an identified history of sexual abuse who also had symptoms of sexual abuse. The results address the extent to which symptoms of sexual abuse in a child psychiatric setting are not identified, nor included in treatment plans and suggest that treatment for this problem is an essential component in the care of any child identified as having this history.

$\mathrm{T}$ HIS STUDY OF THE PREVALENCE OF CHILDREN with a history of sexual abuse hospitalized in the psychiatric setting began when two psychiatric mental health nurses working in an inpatient child psychiatric setting became aware that many of the children had a history of sexual abuse or were displaying symptoms of sexual abuse. These same children were exhibiting a particular set of behaviors that were not acceptable to the general public and, therefore, not well tolerated even in the hospital setting. Some of the behaviors observed were (a) sexual acting out, (b) physical aggression, (c) excessive masturbation, (d) emotional withdrawal, (e) low self-esteem, (f) frequent physical complaints, (g) drop in school performance, and (h) sleep disturbance.

It was also noted that these children were not being assessed or treated for the symptoms of sexual abuse that they displayed. Discussion among the treatment team members revealed a controversy about whether the symptoms the children were displaying were due to their history of sexual abuse. Some treatment team members did not think that current research supported the theory that the symptoms the children were displaying were due to having been sexually abused.

Reprint requests: Patricia Morris, 409 Pauline Blvd., Ann Arbor, MI 48103 .

Accepted for publication December 19, 1990.
From the Child/Adolescent Psychiatric Hospital, University of Michigan, Ann Arbor, Michigan

In response to this dilemma, a study of a clinical population of psychiatrically hospitalized children was conducted to verify the nurses' professional assessment that a high percentage of psychiatrically hospitalized children have a history of sexual abuse and are displaying symptoms of sexual abuse.

\section{Review of the Literature}

In reviewing the current literature on sexual abuse, it was noted that an average of $16 \%$ of the boys and $48 \%$ of the girls (4-12 years of age) in the inpatient psychiatric setting have been sexually abused, while in the general population it is estimated that $10 \%$ of the boys and $20 \%$ of the girls have been sexually abused (Kohan, Pothier, \& Norbeck, 1987). The literature review also revealed a wide variance related to the percentage of children with a history of sexual ab'sse hospitalized in the psychiatric setting. This variance was due to: (1) the definition of child sexual abuse sometimes being limited to only incestuous relationships, (2) incestuous relationships being limited to intercourse only, (3) sexual abuse being limited to intercourse only, (4) underreporting of sexual abuse by abuse victims due to the secrecy surrounding it, fear of being harmed by the abuser, or fear of the abuser harming someone else in the family, and (5) health professionals not asking psychiatrically hospitalized patients if they have been sexually abused (Craine, et al., 1988; Kohan, Pothier, \& Norbeck, 1987; Husain \& Chapel, 1983; Rosenfeld, 1979). Kohan, et al.'s (1987) findings in a study of 4-12-year-old children in 110 inpatient psychiatric hospitals were most similar to that of the population on the child psychiatric inpatient unit in this study.

Frequently, professionals on psychiatric treatment teams diagnose symptoms the child with a history of sexual abuse displays under the following diagnostic categories: attention- 
deficit disorder, conduct disorder, depression, or some other DSM-III-R diagnoses (Lowery, 1987). In American society, children are considered asexual. There is denial that adults may have sexual feelings towards children and may choose to act on these feelings by having sex with a child.

This clinical study was primarily focused on sexual abuse in hospitalized children. Information also was gathered on the prevalence of physical abuse of these children.

\section{Method}

\section{Subjects}

The sample for this study was obtained from the records of 100 children hospitalized in a children's psychiatric hospital in the Midwest over the past five years. The usual census of the unit was 12 children, ranging in age from 4 to 12 years. The length of stay on the unit was approximately 4-12 weeks, and the approximate number of admissions per year was 50 . The diagnoses of the children were widely varied (e.g., attention deficit disorder, pervasive developmental delay, conduct disorder, oppositional disorder, depression, dysthymia, and autism).

\section{Procedures}

A checklist of symptoms commonly exhibited by children with a history of child sexual abuse based on the clinical reports and research findings of Burgess \& Hartman (1986), Burgess (1986), Alterman, Sgroi, \& Roane (1984), and Foley \& Davies (1983) was developed for this study. Burgess and Hartman (1986) studied the phases of sexual abuse and recovery from sexual abuse. They noted what symptoms of the sexual abuse experience were acute and what symptoms were chronic. Alterman, Sgroi, and Roane (1984) reported the best methods for interviewing children who were displaying symptoms of sexual abuse and what symptoms were indicators of sexual abuse. Foley and Davies (1983) reported clinical symptoms of sexual abuse when the victim had not been treated for the abuse. The checklist was used to identify children displaying symptoms of sexual abuse. The checklist included 49 items identified from the literature review, (e.g., body image problems, hurts self physically).

A total of 100 closed records were used as the sample. Systematic sampling was used to determine which children's records of the total number of records would be used. The total number of children admitted over the past 5 years ( $\mathrm{n}=563$ ) was divided by one hundred; this number was used as the nth number. Every nth number then was chosen for the sample.

The client characteristic tool (which gathered data on age, race, history of sexual abuse, etc.) and the checklist of symptomatology of child sexual abuse (which noted symptoms displayed by the child which were indicators of sexual abuse) were then filled out by the two psychiatric-mental health nurses for each of the 100 records in the sample. Since this was a retrospective analysis of data from records of children who had been admitted for psychiatric care in the children's psychiatric hospital, no active contact with human subjects was made and no risk to human subjects was involved. Confidentiality was addressed by not identifying the name, address, phone number, or case record number of any of the patients whose records were reviewed.

\section{Analytic Procedures}

Descriptive statistics were used to describe the sample. The prevalence of symptoms among children with a documented history of child sexual abuse and of children without a documented history of child sexual abuse were determined by frequency counts associated with each group. Tests of associations were used to determine if the type and frequency of diagnoses among subjects were associated with a history of child sexual abuse, and whether this life event was documented or not in the record.

In addition, children having more than 10 symptoms present from the checklist were classified as having prevalent sexual abuse symptomatology. More than 10 symptoms was hosen as the number for prevalence of symptoms of child sexual abuse because mean number of symptoms displayed for children with a history of documented sexual abuse was 20.6 , with a range from 11 to 29 symptoms. More than 10 symptoms was below the mean but was the low end of the range for those having a documented history of child sexual abuse. Also, from a clinical standpoint, if the number of symptoms was too high, children without a documented history of child sexual abuse would be less likely to be included, because they would not specifically have been interviewed for sexual abuse symptomatology.

Since this is a cross-sectional (prevalence) study, associations were examined using chi-square type statistics, or Fisher's Exact test. For continuous outcome measures, Student's t-test or the overall F-test from an ANOVA, were employed to detect outcome differences between or across factor means. For significant F-tests, the multiple comparison procedures suggested by Tukey (for all pairwise comparisons) and Scheffe (for all possible comparisons) were used to investigate different linear contrasts to quantify the effect. Pearson's zero order correlation coefficient was used when examining the association between two continuous variables.

\section{Results}

This sample of 100 closed records of children psychiatrically hospitalized in a Midwest facility over the past 5 years was predominantly male $(\mathrm{n}=83,83 \%)$ and white $(\mathrm{n}=88$, $88 \%)$. The mean age for the sample was 9.27 years $(+/$ -2.474 years) with the median age being 9.00 years and range of 4-12 years old. Most of the children lived with 
either both biological parents $(n=33,33 \%)$ or with only their mother $(n=30,30 \%)$. Thirteen percent $(n=13)$ of the children lived either in foster homes or with adoptive parents. Only $36 \%(\mathrm{n}=36)$ of the children had parents who had always been married.

\section{Prevalence of Documented Sexual Abuse}

Of the 100 records sampled, $31 \%(n=31)$ had a documented history of sexual abuse, and $33 \%(\mathrm{n}=33)$ had been physically abused. The remaining 36 children had no documentation of any abuse.

Of the 100 records sampled, $69 \%$ did not have a documented history of sexual abuse. The records for these children were then examined for the presence of specific sexual abuse symptoms using the Symptomatology of the Sexually Abused Child Checklist (Table 1). Analysis revealed the mean number of symptoms reported was 15.4 $(+1-.656)$. The median was 17 symptoms with the number of symptoms ranging from 2 to 29 . Using above 10 symptoms as the score for displaying prevalent sexual abuse symptomatology, $73 \%(n=73)$ of the sample displayed prevalent symptoms of sexual abuse.

Among the three documented groups, the mean number of symptoms displayed varied significantly $(F=35.58$, $p=.000$ ) with the following appearing among the three groups: no abuse $(\overline{\mathrm{X}}=10.2778 ; \mathrm{SD}=6.264$; range $=$ 2-27), physical abuse $(\overline{\mathrm{X}}=16.0909 ; \mathrm{SD}=4.440$; range $=$ $6-24)$, and sexual abuse $(\bar{X}=20.6129 ; S D=3.887$, range $=8-29$ ).

The distribution of prevalent sexual abuse symptomatology was significantly different across the documented sexual abuse groups $\left(\mathrm{X}^{2}=40.0269, p=.000\right)$ with virtually all abused subjects displaying more than 10 sexual abuse symptoms. Of the 36 subjects with no history of documented sexual or physical abuse, $23(64 \%)$ displayed 10 or less sexual abuse symptoms. The other 13 subjects in the group with no documented history of being sexually or physically abused displayed prevalent sexual abuse symptomatology.

The most prevalent medical diagnoses reported were parent-child problems $(n=30)$, conduct disorder $(n=30)$, and attention deficit disorder with hyperactivity $(n=25)$. Only one medical diagnosis, conduct disorder, appeared to be significantly related to abuse status, suggesting that this diagnosis is more prevalent among sexually abused subjects than nonabused subjects $\left(\chi^{2}(1)=5.419, p=0.0199\right)$. However, this significance is lost when the abuse groups are collapsed.

The common nursing diagnoses made were behavior dangerous to self $(n=33)$, oppositional and aggressive $(n=24)$, and alteration in attention $(n=19)$. Two nursing diagnoses, behavior dangerous to self, and sexual acting out, were more prevalent among those subjects having documented sexual abuse, but this finding was not found to be statistically significant.
TABLE 1. Symptomatology of the Sexually Abused Child Checklist

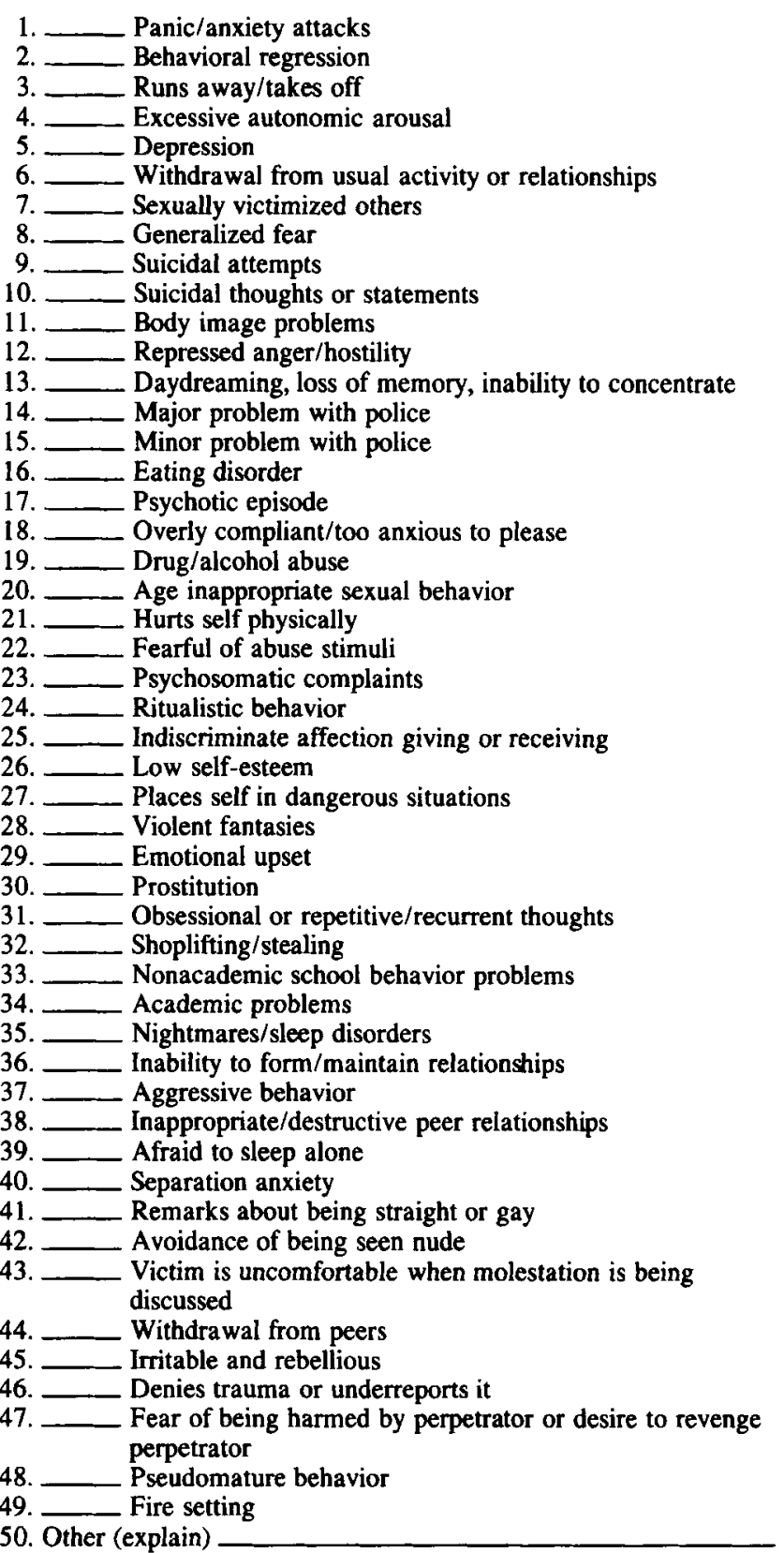

Physicians addressed sexual abuse in the treatment plan of eight of 31 subjects with a documented history of sexual abuse. In five of the cases, treatment was referral of the subject to Protective Services. In two treatment plans, the physician specified that the child be observed for symptoms of sexual abuse, and in one treatment plan, it was specified that treatment be deferred stating that the ego structure of the child was too fragile.

Nurses addressed sexual abuse in the nursing treatment 
plan of three of 31 subjects with a documented history of sexual abuse. The nursing treatment plan in these three cases was to prevent the child from sexually abusing other children and to stop the child from masturbating in public.

There were no cases in which physicians or nurses addressed treatment of sexual abuse in the treatment plan for children with prevalent symptoms of child sexual abuse but with no documented history of child sexual abuse.

\section{Discussion}

The results of this study supported the clinical observation that a significant portion $(n=31)$ of the child population, 4-12 years old, hospitalized in the child psychiatric setting, has a documented history of sexual abuse. In addition, the total percentage of abuse in the child population rose to $64 \%$ $(n=64)$ when children with a documented history of physical abuse $(n=33)$ were added. Analysis of subjects with no documented history of any type of abuse revealed $33 \%$ $(n=13)$ had prevalent symptoms of sexual abuse. Analysis indicated a total of $77 \%(n=77)$ of the population had a history and/or prevalent symptoms of abuse as reported in their records.

Kohan, Pothier \& Norbeck studied 4-12-year-olds in 110 child psychiatric hospitals using record surveys filled out by the supervisors in the individual hospitals. Their results were reported only in percentages. They found that $16 \%$ of the male children in the study had a history of sexual abuse, while this study found that $26 \%$ of the male children had a history of sexual abuse. Kohan and her colleagues found that $48 \%$ of the female children had a history of sexual abuse, while $55 \%$ of the female children in this study had a history of sexual abuse. The percentages may be higher in this study since the chart reviews were done by the two psychiatricmental health nurses, while Kohan and her colleagues had to depend on 110 different supervisors to collect their data.

When examining the question of medical diagnosis for its association with a history of sexual abuse, the only DSMIII-R diagnosis that proved to be significant for those with a documented history of sexual abuse as compared with those without a documented history of sexual abuse, was conduct disorder. Finding that the medical DSM-III-R diagnosis was significant was a surprise, but when looking at the criteria for giving a diagnosis of conduct disorder many symptoms of sexual abuse are found (e.g., sexually abusing others, aggression toward others, running away from home, getting in trouble with the police, cruelty to animals). The diagnosis of conduct disorder is consistent with Burgess and Hartman's (1984) finding that one outcome of child sexual abuse is identification with the offender in which the victim exhibits antisocial behavior and aggressively acts out. This link, however, was not made by the treatment team. None of the nursing diagnoses were statistically significant for a difference between those children with and without a documented history of child sexual abuse.
In discovering whether or not there was an association between history of sexual abuse and treatment plans addressing specific treatment for sexual abuse, this study found that only eight of the 31 physician treatment plans for children with a documented history of child sexual abuse specifically addressed the treatment needs of the sexually abused child. However, these treatment plans were superficial. For example, they either made a referral to protective services $(n=5)$, requested observation for symptoms of abuse $(n=2)$, or requested that the subject of abuse not be brought up because the treating physician believed that the child's ego was too fragile to cope with this approach $(n=1)$. None of the children with prevalent symptoms of sexual abuse and no documented history of sexual abuse had a treatment plan that specified treatment regarding symptoms of sexual abuse. Only three of the 31 nursing treatment plans for children with a documented history of child sexual abuse referred to the sexual abuse. These plans included: (1) close observation to prevent the child from victimizing other children; and (2) teaching the child it was inappropriate to masturbate in public. The authors found this result disturbing, for even in documented cases, nurses were not intervening. In addition, none of the children with prevalent symptoms of sexual abuse and no documented history of sexual abuse had any nursing treatment plan regarding sexual abuse, despite their symptom pattern.

The American Nurses' Association's Standards of Child and Adolescent Psychiatric and Mental Health Nursing Practice (1985) stated that "The nurse uses psychotherapeutic interventions to assist children or adolescents and families to develop, improve, or regain their adaptive functioning, to promote health, prevent illness, and facilitate rehabilitation." (p. 14). The psychiatric nurse helping the child recover from the trauma of sexual abuse has a definite role in assisting the child to regain adaptive functioning.

Current literature supports the fact that sexually abused children need treatment for having been sexually abused (Walker, 1988; Burgess, McCausland \& Wolbert, 1981; Haugarrd, 1988; Giaretto, 1981). Examples of treatment that can be used while the child is psychiatrically hospitalized include play therapy, drawing/art therapy, conventional treatment techniques focused on the sexual abuse issues, and group or family therapy. Play therapy. is a means of working with sexually abused children to allow them to express the abuse experience, and the anger associated with abuse. Play therapy also provides a cognitive structure to relearn power and control and to develop new behavioral skills (Walker, 1988). Art therapy can be a means of understanding the child's world and the trauma the child has experienced (Burgess, McCausland \& Wolbert, 1981). Drawing is a nonverbal way that the child can communicate since some children find it difficult and painful to verbally express what has happened to them. If the child is cognitively able, conventional treatment techniques can be used, with a focus 
TABLE 2. Distribution of Demographics by Documented Sexual Abuse

\begin{tabular}{|c|c|c|c|c|c|c|c|c|}
\hline \multirow[b]{2}{*}{ Variable } & \multirow[b]{2}{*}{$p$-value } & \multicolumn{3}{|c|}{ Documented SA } & \multirow[b]{2}{*}{ Total } & \multirow[b]{2}{*}{$p$-value } & \multirow[b]{2}{*}{$\overline{\mathbf{X}}_{\text {NSAsymp }}$} & \multirow[b]{2}{*}{$S D_{\text {NSAsymp }}$} \\
\hline & & No & Yes & PA & & & & \\
\hline \multicolumn{9}{|l|}{ Gender } \\
\hline Male & $112 \dagger$ & 32 & 22 & 29 & 83 & $.099 *$ & 14.928 & $(6.607)$ \\
\hline $\begin{array}{l}\text { Female } \\
\text { Race }\end{array}$ & & 4 & 9 & 4 & 17 & & 17.706 & $(5.977)$ \\
\hline \multicolumn{9}{|l|}{ Race } \\
\hline $\begin{array}{l}\text { White } \\
\text { Nonwhite }\end{array}$ & $.388 \dagger$ & 34 & 25 & 29 & 88 & $.033^{*}$ & 15.023 & $(6.579)$ \\
\hline \multicolumn{7}{|l|}{ Married } & 16.900 & $(5.587)$ \\
\hline Yes & $002+$ & 21 & 4 & 11 & 36 & $.001^{*}$ & 12.694 & $(6.840)$ \\
\hline No & $.002 T$ & 15 & 27 & 22 & 64 & & 16.922 & $(5.924)$ \\
\hline \multicolumn{9}{|l|}{ Foster } \\
\hline Yes & $.244 \dagger$ & 2 & 5 & 6 & 13 & $.245^{*}$ & 17.385 & $(5.867)$ \\
\hline No & & & & & & & 15.103 & $(6.637)$ \\
\hline Number of symptoms & & $\begin{array}{c}10.278 \\
(6.264)\end{array}$ & $\begin{array}{c}20.613 \\
(3.887)\end{array}$ & $\begin{array}{l}16.091 \\
(4.440)\end{array}$ & $\begin{array}{c}15.400 \\
(6.650)\end{array}$ & & $0.000 \ddagger$ & \\
\hline Age & & $\begin{array}{c}9.444 \\
(2.580)\end{array}$ & $\begin{array}{c}9.182 \\
(2.767)\end{array}$ & $\begin{array}{c}9.161 \\
(2.015)\end{array}$ & $\begin{array}{c}9.270 \\
(2.474)\end{array}$ & $.871 \neq$ & & \\
\hline
\end{tabular}

*For discrete variable analysis a chi-square statistic was employed.

$\dagger$ For testing differences between two group means a Student's t-test was used.

¥To test differences between more than two means an ANOVA procedure was employed with the resulting test statistics being an overall F-test SA, Sexual abuse; PA, Physical abuse; NSAsymp, Number of sexual abuse symptoms.

on the child's anger, learned sexualized behavior, trust and safety issues, fear generated by abuse and self image disturbance, as proposed by Haugarrd (1988). Another approach is a program that includes individual, group, and family treatment not only to treat the sexually abused child but the child's family as well (Giaretto, 1981).

As a direct result of this study, a change in the nursing data base assessment tool used to interview parents and children admitted to the child psychiatric inpatient unit was facilitated. The new data base includes a section that asks: (a) What concerns do you have about your child's behavior sexually? (b) Is there a possibility that your child has been sexually abused? (c) Do you have any concerns regarding your child's sexual education? (d) Can we assist you in providing sexual education for your child? Further, if there is a concern that the child may have been sexually abused, a more complete individual assessment devised by the child's treatment team will be undertaken by the members of the team.

The results of this study have been shared with the nurses and physicians on the child treatment teams. An educational treatment group for children from dysfunctional or abusive families and an education and support group for the parents of the 4-12-year-old children admitted to the inpatient child psychiatric hospital have been initiated on this child psychiatric inpatient unit.

\section{Limitations of the Study}

Certain limitations were inherent in this study from the beginning such as limiting data collection to one inpatient setting. Therefore, the findings may not be generalizable to children in other child psychiatric hospitals. Also, this study only dealt with the closed records of the 100 subjects and therefore was limited to the type and amount of information that was available in the record. This is a limitation since often the children had not been interviewed about abuse. Therefore, details and symptoms related to child sexual abuse often were not present in the record. Further research replicating this study and using the tools developed by the authors would be useful to ensure the validity and reliability of the tools and to verify the results of the study.

\section{Summary}

There is an inadequate level of assessment and treatment by professionals who interview children and their parents in the child psychiatric setting to identify children who experience actual or potential health problems resulting from child sexual abuse. Diagnoses of children with a documented history of child sexual abuse or prevalent symptoms of child sexual abuse do not reflect the fact that the child is suffering from the sequela of having been sexually abused. Nonrecognition of child sexual abuse symptoms among the hospitalized child population leads to lack of treatment for child sexual abuse. This study validates the need for more aggressive, specific assessments for child sexual abuse that will ultimately lead to appropriate treatment and healing.

\section{References}

Alterman, B., Sgroi, S. \& Roane, T . (1984). Handout courtesy of District III, Child Protection Team, University of Florida, a Department of 
Pediatrics Program, sponsored by Health and Rehabilitative Services/Children's Medical Services.

Burgess. A.W. . McCausland. M.P. \& Wolpert. W.A. (1981). Children's drawings as indicators of sexual trauma. Perspectives in Psichiatric Care, $X I X(2), 50-58$.

Burgess, A.W. . \& Hartman. C.R. (1986). Child sexual abuse: Generic root of the victim experience. Journal of Psychotherapy and the Family, 2(2), $83-92$

Burgess, A.W. ( 1986). Youth at rish: Understanding runaway and exploited youth. Washington. DC: National Center for Missing and Exploited Children.

Craine. L. . Henson. C. . Colliver, J. \& \& Mac Lean. D. (1988). Prevalence of a history of sexual abuse among female psychiatric patients in a state hospital system. Hospital and Community Psichiatry. 39(3). $300-304$.

Fredrick. C. (1986). Post traumatic stress disorder and child molestation. Sexual medicine. New York: Prager

Foley. T. \& Davies. M. (1983). Rape: Nursing care of victims. St. Louis: C.V. Mosby Co.
Giaretto. H. (1981). A comprehensive child sexual abuse treatment program. Child Abuse and Neglect, 6;263-278.

Haugaard. J. (1988). The sexual abuse of children: A comprehensive guide to current knowledge and intervention strategies. San Francisco: Jossey-Bass Publishers

Hausain. A.. \& Chapel. J. (1983). History of incest in girls admitted to a psychiatric hospital. American Journal of Psychiatry, 138: 967-970.

Kohan. M. . Pothier. P., \& Norbeck, J. (1987). Hospitalized children with history of sexual abuse: Incidence and care issues. American Journal of Orthopsychiatry, 57(2), 258-264.

Lowery. M. (1987). Adult survivors of childhood incest. Journal of Psychosocial Nursing, 25(2), 27-41.

Rosenfeld. A. (1979). Incidence of history of incest among 18 female psychiatric patients. American Journal of Psychiatry, 136, $791-795$.

Walker. L. (1988). Handbook on sexual abuse of children: Assessment and treatment issues. New York: Springer Publishing Co. 